\title{
Income and Education Inequalities in Brain and Central Nervous System Cancer Incidence in Canada: Trends over Two Decades
}

\author{
Alysha Roberts ${ }^{1}$, Min $\mathrm{Hu}^{2}$, Mohammad Hajizadeh ${ }^{2}$ \\ ${ }^{1}$ Faculty of Medicine, Dalhousie University, ${ }^{2}$ School of Health Administration, Faculty of Health, Dalhousie University, \\ Halifax, NS, Canada
}

The socioeconomic gradient of brain and central nervous system (CNS) cancer incidence in Canada is poorly understood. This study aimed to measure socioeconomic inequalities in brain and CNS cancer incidence in Canada from 1992 to 2010. Using a unique census division level dataset $(n=280)$ pooled from the Canadian Cancer Registry $(C C R)$, the Canadian Census of Population and the National Household Survey, we measured brain and CNS cancer incidence in Canada. The age-adjusted concentration index (C) was used to measure income- and education-related inequalities in brain and CNS cancers in Canada, and for men and women, separately. Time trend analyses were conducted to examine the changes in socioeconomic inequalities in brain and CNS cancers in Canada over time. The results indicated that the crude brain and CNS cancer incidence increased from 7.29 to 8.17 per 100,000 (annual percentage change: 0.70 ) over the study period. The age-adjusted $C$ results suggested that the brain and CNS cancer incidence was not generally significantly different for census division of different income and educational levels. There was insufficient evidence to support changes in income and education-related inequalities over time. Since the incidence of brain and CNS cancers in Canada showed no significant association with socioeconomic status, future cancer control programs should focus on other risk factors for this cancer subset.

Key Words Social inequalities, Brain neoplasms, Central nervous system neoplasms, Incidence, Canada

\section{INTRODUCTION}

While primary cancers of the central nervous system (CNS) are rare, both benign and malignant brain tumors result in high morbidity and mortality worldwide [1,2]. Recent studies suggest that globally, CNS cancers are becoming more prevalent [3]. In Canada, brain cancer represents the highest proportion of cancer deaths among younger adults and are highly morbid across the lifespan [4]. Environmental factors such as exposure to ionizing radiation and certain occupational hazards have an established relationship with the incidence of CNS cancers [5-8]. However, there remains a lack of evidnce supporting relationships with social determinants of health such as income and education.

To date, some international studies have examined the association between socioeconomic status (SES) and the incidence of cancers originating from the CNS. These studies have yielded mixed findings. A study by Quinn and Babb [9] found the highest income quintile compared to the lowest income quintile in England and Wales had a 25\% higher incidence of brain tumors. In their 2015 study on glioblastoma multiforme, the most common form of brain cancer, Muquit et al. [10] used average weekly household income, percentage of unemployment, population density, indices of deprivation and percentage of households with no car as SES measures and found that a higher incidence of glioblastoma existed among those in districts with higher average weekly income and lower unemployment. This positive relationship between higher SES and incidence of brain tumors is consistent with some of the existing literature $[9,11,12]$. In contrast, other studies suggest that there is no correlation between higher SES and the incidence of brain tumors [13].

Although the current studies highlight the socioeconomic gradient in some types of cancer in Canada [14-23], no study

Received March 1, 2021, Revised May 21, 2021, Accepted June 9, 2021

Correspondence to Mohammad Hajizadeh, E-mail: m.hajizadeh@dal.ca, https://orcid.org/0000-0002-4591-8531

Check for updates 
quantifies socioeconomic inequalities in the incidence of brain and CNS cancer in Canada. Using the Canadian Cancer Registry (CCR), Canadian Census of Population (CCP), and National Household Survey (NHS) this study fills this gap in the literature by examining income and education inequalities in the incidence of all malignant brain and central nervous system tumors over time from 1992 to 2010 . This is the period that the CCR data available for all provinces in Canada at the Statistics Canada's Research Data Centres. The results of this study contribute to our understanding of the SES gradient in brain and CNS cancer incidence in Canada over the past two decades.

\section{MATERIALS AND METHODS}

\section{Data}

Data were obtained from the CCR, the CCP (1992, 1996, 2001, 2006) and the NHS (2011). The CCR contains information about the incidence of all new cancer cases since 1992 in all provinces and territories in Canada. Data from ten Canadian provinces were included in the study: Newfoundland and Labrador (NL), Prince Edward Island (PE), Nova Scotia (NS), New Brunswick (NB), Quebec (QC), Ontario (ON), Manitoba (MB), Saskatchewan (SK), Alberta (AB), and British Columbia (BC). Territories were not included in the analysis due to the lower number of brain and CNS cancer incidences reported in each year.

The CCR was used to obtain basic demographic information about those diagnosed with brain and CNS cancer and a six-digit postal code corresponding to their location of permanent residence. The Postal Code Conversion File plus Version D software was used to find the Census Division (CD) coordinates of each patient diagnosed with brain and CNS cancer based on their six-digit postal codes in the CCR. The CDs are defined as a group of neighboring municipalities joined together for regional planning and management [24]. Based on this information, the number of new cases of brain and CNS cancer in each CD was calculated.

The CCR does not collect information on the SES of patients. Thus, the CCP and NHS datasets were used to construct a new dataset containing SES (average and median equivalized household income and proportion of individuals with a bachelor's degree or above) and population demographics for each CD in Canada. Based on CD coordinates, the CCP/NHS data were linked to the CCR data as follows: CCP 1992 to CCR 1992-1993, CCP 1996 to CCR 19941998, CCP 2001 to CCR 1999-2003, CCP 2006 to CCR 2004-2008, and NHS 2011 to CCR 2009-2010. Since the 2011 CCP does not contain SES information for its respondents, the $2011 \mathrm{NHS}$ was used to obtain relevant income and education data for 2009 and 2010. The linked dataset contained the number of brain and CNS cancer diagnoses, SES, and population characteristics at the CD-level $(n=280)$ over the period between 1992 and 2010.

\section{Measures}

The outcome of interest in this study was brain and CNS cancer incidence. Brain and CNS cancer was defined based on the International Classification of Diseases for Oncology third edition codes C70.0 to 72.9. Average and median household income level and proportion of individuals with a bachelor's degree or above were used as three SES variables in the study. Income and education levels for each CD was obtained from the CCP/NHS database. Annual household income was equivalized as per the Organization for Economic Co-operation and Development (OECD) publications to adjust the household size [25]. This involved dividing household size by the square root of household size when we estimated the average and median household income for each CD.

\section{Statistical analysis}

\section{Measuring socioeconomic inequalities}

The concentration index (C) approach was used to quantify the degree of socioeconomic inequality in brain and CNS cancer incidence. The $\mathrm{C}$ was measured based on the concentration curve and the line of perfect equality, which represents an equal distribution of disease burden across all levels of SES. The concentration curve plots the cumulative incidence of brain and CNS cancer (y-axis) against the cumulative proportion of the population in ascending order of SES. The $\mathrm{C}$ is computed as twice the area between the concentration curve and the line of perfect equality. A negative value of the $\mathrm{C}$ indicates a disproportionate concentration of brain and CNS cancer incidence among those of lower SES, while a positive value suggests an increased incidence of brain and CNS cancer among those of higher SES. The $C$ ranges from -1 to 1 , with 0 representing perfect equality.

The following "convenient regression" formula can be used to estimate the C [26]:

$$
2 \sigma_{r}^{2}\left(\frac{B C I_{i}}{\mu}\right)=\alpha+\delta r_{i}+\varepsilon_{i}
$$

where $\mathrm{BCl}_{i}$ shows $\mathrm{CD} i$ 's brain and CNS cancer incidence rate, $\mu$ is the mean incidence rate for brain and CNS cancer for all CDs, $\alpha$ is the intercept. The $r_{i}$ is the CD $i$ 's fractional rank in the SES distribution, which is calculated as $r_{i}=i / n$, with $i$ $=1$ for the lowest SES and $i=n$ for the highest SES. The $\sigma_{r}^{2}$ denotes the variance of $r_{i}$. The ordinary least squares (OLS) estimate of $\delta$ in Equation 1 and its standard error demonstrates the value and the standard error for the crude $\mathrm{C}$, correspondingly. The age-adjusted (also called age-standardized) socioeconomic-related inequality can be calculated using an indirectly standardized concentration index by including the age variable in Equation 1 as follows [27]:

$$
2 \sigma_{r}^{2}\left(\frac{B C I_{i}}{\mu}\right)=\alpha+\gamma r_{i}+\beta A g e_{i}+v_{i}
$$

where $A g e_{i}$ indicates the average of individuals in the CD 
$i$ and $\beta$ is the corresponding coefficient for $A g e_{i}$. The OLS estimate of $\gamma$ in Equation 2 is the age-adjusted $C$.

The age-adjusted $C$ was calculated according to three measures of SES (i.e., average and median equivalized household income and proportion of the population with a bachelor's degree or above) for the period between 1992 and 2010 to determine income- and education-related inequalities in brain and CNS cancer incidence. Comparisons were made for the total population and for males and females, separately. The total number of relevant populations in each CD was used to calculate the incidence of brain and CNS cancer as well as the weight in the calculation of the $C$ in each year.

\section{Measuring trends in the incidence and socioeconomic inequalities}

Poisson regression was conducted to calculate annual percent change in brain and CNS cancer incidence over the study period. Trend analyses for income- and education-related inequalities were performed by plotting the age-adjusted $C$ on the $y$-axis against time (19 points corresponding to the years from 1992 to 2010 ) on the $x$-axis. The negative (positive) coefficient for the time indicates an increase in the concentration of brain and CNS cancer incidence among low (high) SES Canadian over time. All the analyses were performed in V.15 of the STATA software package (StataCorp, College Station, TX, USA).

\section{Ethics approval}

This article does not contain any studies with human participants or animals performed by any of the authors. All data used in this study were accessed from Statistics Canada's Research Data Centre. Statistics Canada ensures ethical handling of all individual- and population-level information. Data accessed through the Research Data Centre is exempt from approval by the Research Ethics Board as per the Tri-council policy statement: Ethical conduct for research involving humans article 2.2 (a).

\section{RESULTS}

\section{Crude brain and CNS cancer incidence}

Figure 1 depicts the crude incidence rate in brain and CNS cancer per 100,000 population in Canada from 1992 to 2010. The total incidence of brain and CNS cancer has trended upwards from 7.29 per 100,000 in 1992 to 8.17 per 100,000 in 2010. The incidence remained higher in males compared to females over the study period. The time trend analysis indicated that brain and CNS cancer diagnoses per 100,000 in Canada have increased by 0.70 percent each year over the study period. For males, incidence per 100,000 increased by 0.76 percent each year, while for females the increase was 0.61 percent per year over the 19-year period studied.

The crude incidence rate in brain and CNS cancer per 100,000 population for each Canadian province can be found

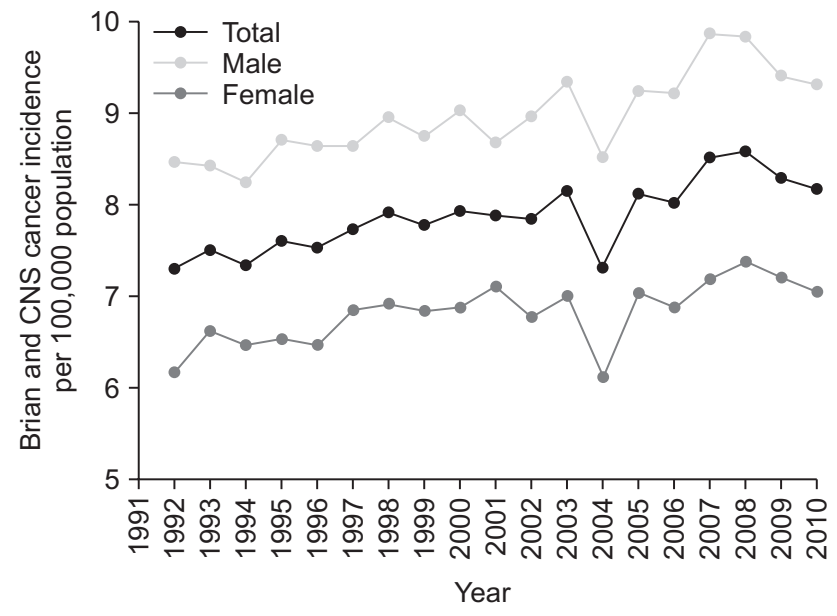

Figure 1. Crude incidence in brain and CNS cancer per 100,000 males, females, and total population of in Canada, 1992 to 2010. CNS, central nervous system.

in Table 1. There was no significant increase in brain and CNS cancer diagnoses over time in most provinces. The time trend analysis indicates the greatest annual percentage change in NL\&PE with 3.8 percent $(P$-value $=0.02)$ from 1992 to 2010.

\section{Socioeconomic Inequalities in brain and CNS cancer incidence}

\section{Income-related inequality in brain and CNS cancer incidence}

Table 2 reports the magnitude of income-related inequalities, the age-adjusted $\mathrm{Cs}$, in brain and CNS cancer incidence over time. Although the $C$ values have been negative over the majority of the nineteen-year period analyzed, income were not consistently associated with the incidence of brain and CNS cancer to a level of statistical significance. Trend analysis of income-related inequality when we used average equivalized income to measure income-related inequalities in brain and CNS cancer did not change for males or females over time $(P$-value $=0.902)$. When we used median equivalized income level in the measurement of income-related inequality, the trend analysis suggested an increase in brain and CNS cancer among those with more income over time $(P$-value < $0.05)$. This pattern was also significant for males alone ( $P$-value $<0.02$ ).

\section{Education-related inequality in brain and CNS cancer incidence}

Table 3 reports age-adjusted education-related inequalities in brain and CNS cancer incidence over time. Education-inequalities were not consistently associated with the incidence of brain and CNS cancer to a level of statistical significance. There were several years in which a significant education-related inequality existed, but no established trend overall. 


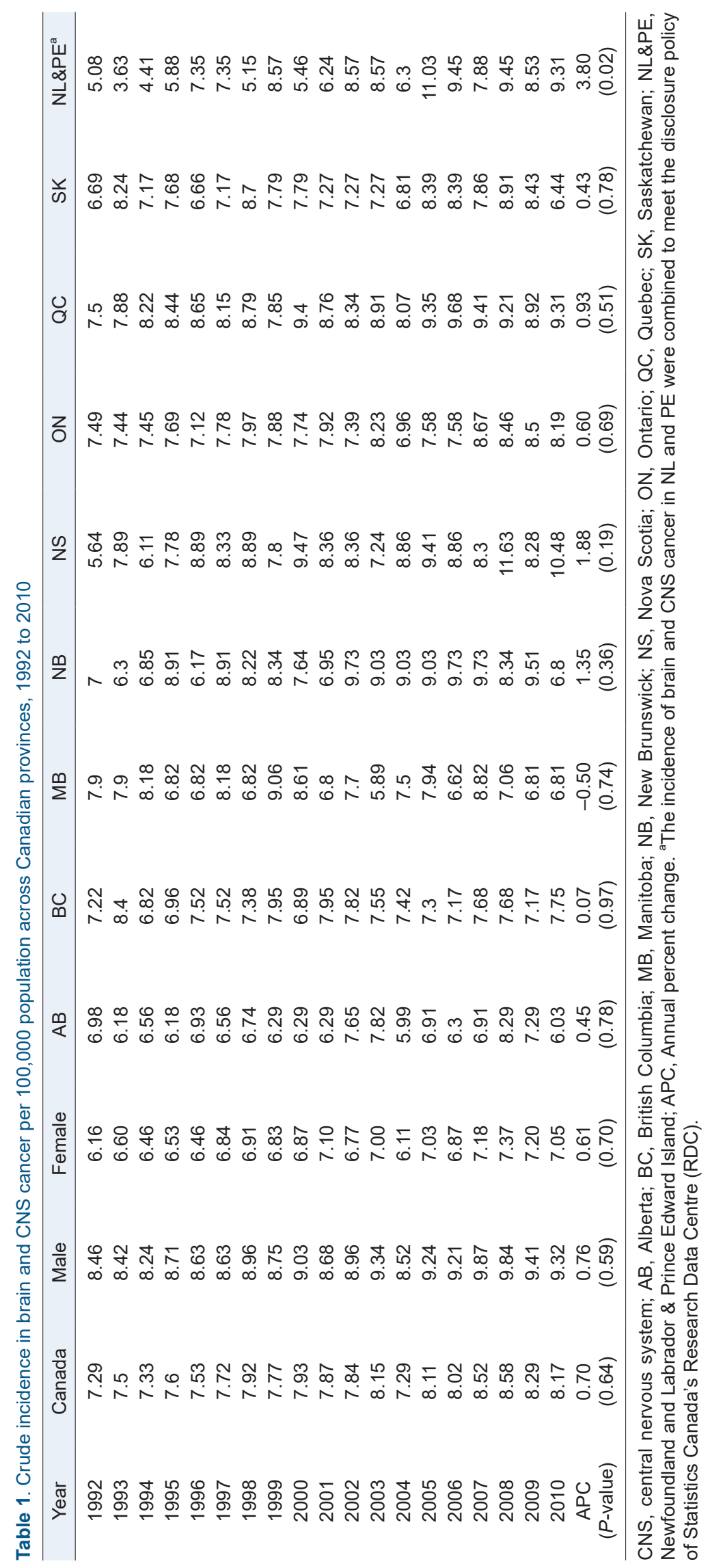


Roberts et al.

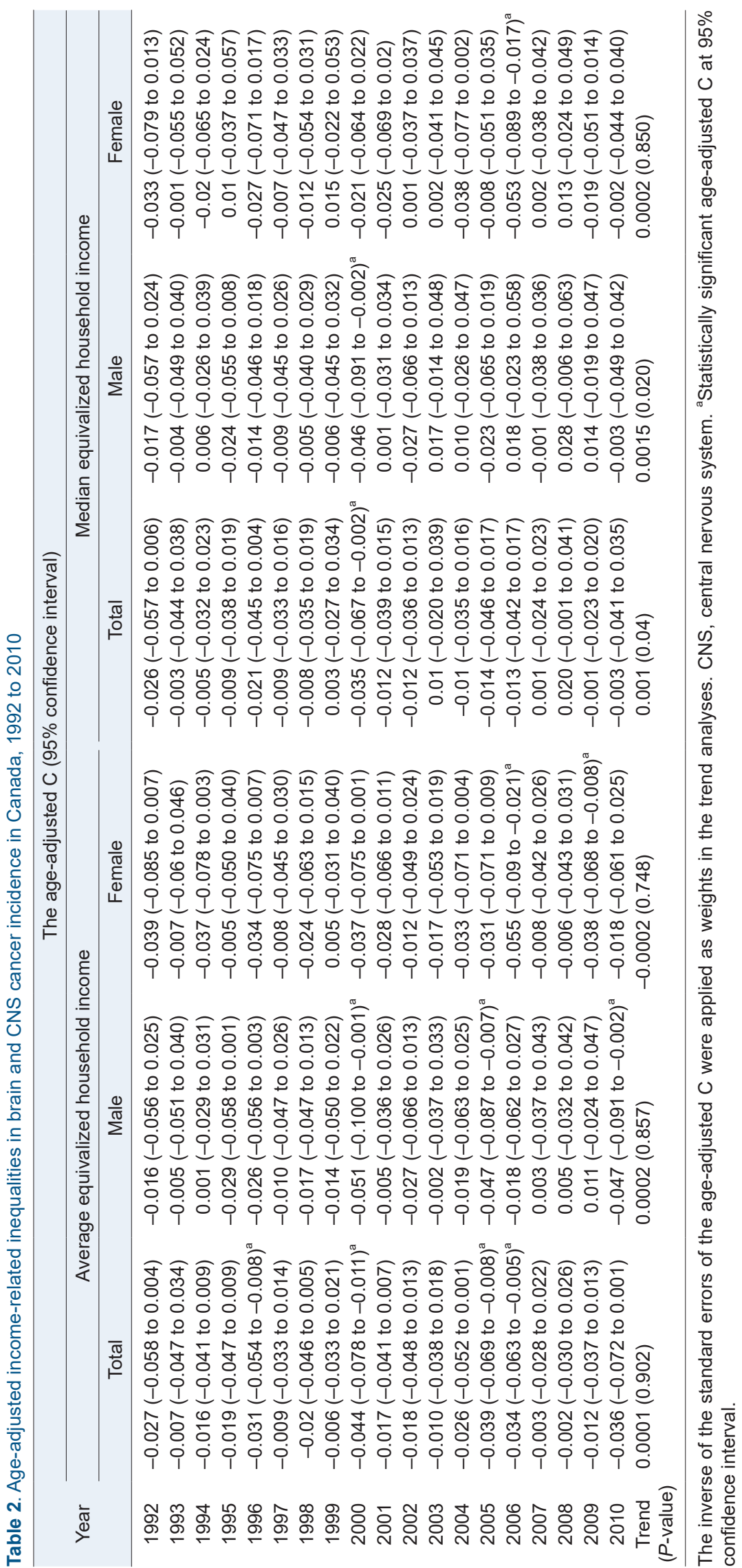


Table 3. Age-adjusted education-related inequalities in brain and CNS cancer incidence in Canada, 1992 to 2010

\begin{tabular}{|c|c|c|c|}
\hline \multirow{2}{*}{ Year } & \multicolumn{3}{|c|}{ The age-adjusted C (95\% confidence interval) } \\
\hline & Total & Male & Female \\
\hline 1992 & $-0.039(-0.073 \text { to }-0.005)^{\mathrm{a}}$ & $-0.031(-0.072$ to 0.009$)$ & $-0.045(-0.093$ to 0.003$)$ \\
\hline 1993 & $-0.01(-0.057$ to 0.037$)$ & $0.016(-0.036$ to 0.068$)$ & $-0.035(-0.101$ to 0.031$)$ \\
\hline 1994 & $-0.03(-0.053 \text { to }-0.007)^{\mathrm{a}}$ & $-0.002(-0.029$ to 0.026$)$ & $-0.061(-0.099 \text { to }-0.023)^{\mathrm{a}}$ \\
\hline 1995 & $-0.009(-0.032$ to 0.013$)$ & $-0.012(-0.037$ to 0.013$)$ & $-0.001(-0.038$ to 0.036$)$ \\
\hline 1996 & $-0.025(-0.045 \text { to }-0.005)^{\mathrm{a}}$ & $-0.022(-0.051$ to 0.007$)$ & $-0.021(-0.055$ to 0.014$)$ \\
\hline 1997 & $0.011(-0.01$ to 0.032$)$ & $0.009(-0.025$ to 0.043$)$ & $0.016(-0.019$ to 0.051$)$ \\
\hline 1998 & $-0.027(-0.049 \text { to }-0.005)^{a}$ & $-0.018(-0.046$ to 0.009$)$ & $-0.035(-0.074$ to 0.003$)$ \\
\hline 1999 & $-0.011(-0.032$ to 0.01$)$ & $-0.012(-0.04$ to 0.016$)$ & $-0.006(-0.04$ to 0.027$)$ \\
\hline 2000 & $-0.029(-0.067$ to 0.009$)$ & $-0.029(-0.082$ to 0.025$)$ & $-0.027(-0.064$ to 0.01$)$ \\
\hline 2001 & $-0.022(-0.045$ to 0.002$)$ & $-0.007(-0.033$ to 0.019$)$ & $-0.034(-0.075$ to 0.008$)$ \\
\hline 2002 & $-0.02(-0.048$ to 0.008$)$ & $-0.008(-0.054$ to 0.038$)$ & $-0.035(-0.066 \text { to }-0.003)^{a}$ \\
\hline 2003 & $-0.035(-0.058 \text { to }-0.012)^{a}$ & $-0.027(-0.055$ to 0$)$ & $-0.041(-0.077 \text { to }-0.005)^{\mathrm{a}}$ \\
\hline 2004 & $-0.025(-0.047 \text { to }-0.004)^{a}$ & $-0.035(-0.07$ to 0.001$)$ & $-0.011(-0.042$ to 0.021$)$ \\
\hline 2005 & $-0.025(-0.057$ to 0.007$)$ & $-0.011(-0.055$ to 0.032$)$ & $-0.04(-0.077$ to -0.003$)$ \\
\hline 2006 & $-0.027(-0.053 \text { to }-0.001)^{\mathrm{a}}$ & $-0.018(-0.052$ to 0.017$)$ & $-0.038(-0.072 \text { to }-0.003)^{\mathrm{a}}$ \\
\hline 2007 & $0.001(-0.021$ to 0.022$)$ & $-0.003(-0.04$ to 0.034$)$ & $0.008(-0.024$ to 0.04$)$ \\
\hline 2008 & $-0.019(-0.043$ to 0.006$)$ & $-0.026(-0.06$ to 0.007$)$ & $-0.007(-0.042$ to 0.029$)$ \\
\hline 2009 & $-0.02(-0.044$ to 0.004$)$ & $-0.016(-0.052$ to 0.02$)$ & $-0.022(-0.054$ to 0.01$)$ \\
\hline 2010 & $-0.042(-0.068 \text { to }-0.016)^{\mathrm{a}}$ & $-0.058(-0.088 \text { to }-0.027)^{\mathrm{a}}$ & $-0.018(-0.048$ to 0.013$)$ \\
\hline Trend ( $P$-value $)$ & $-0.0003(0.677)$ & $-0.0013(0.112)$ & $0.0007(0.403)$ \\
\hline
\end{tabular}

The inverse of the standard errors of the age-adjusted $\mathrm{C}$ were applied as weights in the trend analyses. CNS, central nervous system. ${ }^{\text {a }}$ Statistically significant age-adjusted $\mathrm{C}$ at $95 \%$ confidence interval.

Results of the trend analysis indicated that there was not a significantly increased incidence of brain and CNS cancer among those with less education compared to those with more education $(P$-value $<0.677)$. There was also no significant time trend when assessed based on gender, although there were more years of significant inequality for females compared to males.

\section{DISCUSSION}

This study aimed to assess income-related and education-related patterns in the incidence of brain and CNS cancer in Canada from 1992 to 2010 . The descriptive results suggested that the crude incidence of brain and CNS cancer has increased over time. The increases in the incidence rate, however, were not statistically significant in most provinces. These results are consistent with the results of some developed countries, which suggest no statistical increase in brain and CNS cancer incidence over a similar time period [28]. The statistical increase in NL\&PE is consistent with a study [29] reporting an increasing incidence of brain cancer in several regions of Spain. Further research is warranted to determine what factors may be contributing to the increase in the incidence of brain and CNS cancer in NL\&PE.

Our results assessing trends in income- and education-related inequalities in brain and CNS cancer generally revealed no significant association. The age-adjusted $C$ results suggested negative income and education gradients in the in- cidence of brain and CNS cancer in Canada. However, the observed income and education-related in brain and CNS cancer were not statistically significant in most years. There is insufficient evidence to suggest any changes in socioeconomic inequalities in brain and CNS cancer. These findings are inconsistent with research from other countries that have found a higher incidence of brain and CNS cancer among those of higher affluence [6,10-12]. This may be partially explained by differences in how socioeconomic variables were operationalized in each study or differences in environmental exposures. Our findings are similar to the study by Nilsson et al. [13] (2018), who similarly used national cancer registry data and population-level statistics to analyze the role of socioeconomic variables in the brain and CNS cancer incidence in Sweden. The authors found no significant relationship between higher income and a higher incidence of brain and CNS cancer.

Additionally, we observed no significant pattern of education-related inequalities in brain and CNS cancer incidence over the study period. This also differs from much of the past literature. In their study of socioeconomic characteristics of patients with glioblastoma multiforme, for example, Muquit et al. [10] (2015) found increased rates of brain and CNS cancer among those with higher education. They posited that those with higher education seek and access medical care earlier and more frequently, leading to increased detection rates. Higher education is also associated with increased health literacy and health behaviors, including accessing medical care 
Roberts et al.

[30]. Our finding of no significant education-inequalities in the incidence of brain and CNS cancer may indicate that these differences are not as common within the Canadian context.

The present study is unique in the literature in that we assessed not only the relationship between brain and CNS cancer incidence and socioeconomic status but also the trends in these relationships over time. However, there were some notable limitations to this study. First, due to the availability of the dataset, SES data from discrete time periods (i.e., CCP years and NHS) were extrapolated to measure socioeconomic inequalities in brain and CNS cancer incidence for multiple years. It is possible that annually updated SES information would display a more robust relationship than can be explored with the present dataset. Third, although some studies indicated that area-based and individual-based estimates of SES are comparable, neighborhood characteristics may not always reflect individual characteristics due to ecological fallacy $[31,32]$. Since both and area- and individual-level SES were shown to be independently correlated with health outcomes [33], future research should focus on the associations between area- and individual-level SES and brain and CNS cancer incidence in Canada. Fourth, we did not measure socioeconomic inequalities in the incidence of specific brain and CNS tumors (e.g., meningioma, glioblastoma), for which different relationships with SES may exist $[11,34]$. Thus, further studies are required to measure the socioeconomic gradients in the incidence of specific brain and CNS tumors.

Given the high mortality rates associated with brain and CNS cancer, it is also crucial to gain a deeper understanding of whether socioeconomic inequalities exist for mortality rates for brain and CNS cancer. It is possible that despite an unclear relationship between brain and CNS cancer incidence and socioeconomic variables, those with higher income and education level are receiving better or earlier treatment that decreases their mortality. Thus, future studies should aim to explore further the pattern of socioeconomic inequalities in brain and CNS cancer mortality in Canada.

\section{ACKNOWLEDGMENTS}

We would like to thank Statistics Canada's Atlantic Research Data Centre at Dalhousie University for facilitating access to the Canadian Cancer Registry, the Canadian Census of Population and the National Household Survey. This research was conducted with the support of the Dalhousie Medical Research Foundation Cresco Studentship and a Research Development Grant of the Faculty of Health at Dalhousie University.

\section{CONFLICTS OF INTEREST}

No potential conflicts of interest were disclosed.

\section{ORCID}

Alysha Roberts, https://orcid.org/0000-0002-8503-0975

Min Hu, https://orcid.org/0000-0002-7839-2812

Mohammad Hajizadeh,

https://orcid.org/0000-0002-4591-8531

\section{REFERENCES}

1. Shah V, Kochar P. Brain cancer: implication to disease, therapeutic strategies and tumor targeted drug delivery approaches. Recent Pat Anticancer Drug Discov 2018;13:70-85.

2. Leece R, Xu J, Ostrom QT, Chen Y, Kruchko C, BarnholtzSloan JS. Global incidence of malignant brain and other central nervous system tumors by histology, 2003-2007. Neuro Oncol 2017;19:1553-64.

3. GBD 2016 Neurology Collaborators. Global, regional, and national burden of neurological disorders, 1990-2016: a systematic analysis for the Global Burden of Disease Study 2016. Lancet Neurol 2019;18:459-80.

4. Canadian Cancer Society. Canadian cancer statistics 2017. Toronto, Canadian Cancer Society, 2017.

5. Preston-Martin S, Reema M, Reema C. Nervous system. In: Schottenfeld D, Fraumeni JF, eds. Cancer Epidemiology and Prevention. 3rd ed. Oxford, Oxford University Press, pp 1173-95, 2006.

6. Rachet B, Mitry E, Quinn MJ, Cooper N, Coleman MP. Survival from brain tumours in England and Wales up to 2001. $\mathrm{Br} J$ Cancer 2008;99(Suppl 1):S98-101.

7. McKean-Cowdin R, Calle EE, Peters JM, Henley J, Hannan $\mathrm{L}$, Thurston GD, et al. Ambient air pollution and brain cancer mortality. Cancer Causes Control 2009;20:1645-51.

8. Smoll NR, Brady Z, Scurrah K, Mathews JD. Exposure to ionizing radiation and brain cancer incidence: the Life Span Study cohort. Cancer Epidemiol 2016;42:60-5.

9. Quinn M, Babb P. Cancer trends in England and Wales, 19501999. Health Stat Q 2000;8:5-19.

10. Muquit S, Parks R, Basu S. Socio-economic characteristics of patients with glioblastoma multiforme. J Neurooncol 2015;125: 325-9.

11. Barker DJ, Weller RO, Garfield JS. Epidemiology of primary tumours of the brain and spinal cord: a regional survey in southern England. J Neurol Neurosurg Psychiatry 1976;39:2906.

12. Porter AB, Lachance DH, Johnson DR. Socioeconomic status and glioblastoma risk: a population-based analysis. Cancer Causes Control 2015;26:179-85.

13. Nilsson J, Holgersson G, Järås J, Bergström S, Bergqvist M. The role of income in brain tumor patients: a descriptive registerbased study: no correlation between patients' income and development of brain cancer. Med Oncol 2018;35:52.

14. Momenimovahed Z, Salehiniya $H$. Epidemiological characteristics of and risk factors for breast cancer in the world. Breast Cancer (Dove Med Press) 2019;11:151-64. 
15. Borugian MJ, Spinelli JJ, Abanto Z, Xu CL, Wilkins R. Breast cancer incidence and neighbourhood income. Health Rep 2011;22:7-13.

16. Hajizadeh M, Johnston GM, Manos D. Socio-economic inequalities in lung cancer incidence in Canada, 1992-2010: results from the Canadian Cancer Registry. Public Health 2020;185:189-95.

17. Willis K, Hajizadeh M. Socioeconomic inequalities in gastric cancer incidence in Canada: 1992-2010. Acta Oncol 2020;59: 1333-7.

18. Ologbenla A, Hu M, Hajizadeh M. Socioeconomic status and incidence of pediatric leukemia in Canada: 1992-2010. Cancer Epidemiol 2019;61:14-22.

19. Densmore R, Hajizadeh M, Hu M. Trends in socio-economic inequalities in bladder cancer incidence in Canada: 1992-2010. Can J Public Health 2019;110:722-31.

20. Charles M, Hajizadeh M. Trends in socioeconomic inequalities in the incidence of cutaneous melanoma in Canada from 1992 to 2010 [published online ahead of print February 22, 2020]. J Public Health. doi: 10.1007/s10389-020-01232-z.

21. Morriscey C, Hajizadeh M. Income and education inequalities in cervical cancer incidence in Canada, 1992-2010 [published online ahead of print August 13, 2020]. J Public Health (Oxf). doi: 10.1093/pubmed/fdaa128.

22. Anthes LE, Hajizadeh M. Socioeconomic inequalities in pancreatic cancer incidence in Canada: evidence from Cancer Registry data [published online ahead of print July 14, 2020]. J Public Health. doi: 10.1007/s10389-020-01360-6.

23. Campbell M, Hajizadeh M. Trends in socioeconomic inequalities in the incidence of ovarian cancer among women in Canada: 1992-2010. Women Health 2021;61:381-92.

24. McDonald JT, Johnson-Obaseki S, Hwang E, Connell C, Corsten $M$. The relationship between survival and socio-economic status for head and neck cancer in Canada. J Otolaryngol Head Neck Surg 2014;43:2.

25. OECD. Growing unequal?: Income distribution and poverty in
OECD countries. Paris, OECD, 2008.

26. Kakwani N, Wagstaff A, van Doorslaer E. Socioeconomic inequalities in health: measurement, computation, and statistical inference. J Econom 1997;77:87-103.

27. O’Donnell O, van Doorslaer E, Wagstaff A, Lindelow M. Analyzing health equity using household survey data: a guide to techniques and their implementation. Washington, DC, World Bank, 2008.

28. Kim SJ, loannides SJ, Elwood JM. Trends in incidence of primary brain cancer in New Zealand, 1995 to 2010. Aust N Z J Public Health 2015;39:148-52.

29. Ugarte MD, Adin A, Goicoa T, Casado I, Ardanaz E, Larrañaga N. Temporal evolution of brain cancer incidence in the municipalities of Navarre and the Basque Country, Spain. BMC Public Health 2015;15:1018.

30. Cohen AK, Syme SL. Education: a missed opportunity for public health intervention. Am J Public Health 2013;103:997-1001.

31. Mustard CA, Derksen S, Berthelot JM, Wolfson M. Assessing ecologic proxies for household income: a comparison of household and neighbourhood level income measures in the study of population health status. Health Place 1999;5:157-71.

32. Krieger N, Chen JT, Waterman PD, Soobader MJ, Subramanian SV, Carson R. Geocoding and monitoring of US socioeconomic inequalities in mortality and cancer incidence: does the choice of area-based measure and geographic level matter?: The Public Health Disparities Geocoding Project. Am J Epidemiol 2002;156:471-82.

33. Buajitti E, Chiodo S, Rosella LC. Agreement between area- and individual-level income measures in a population-based cohort: Implications for population health research. SSM Popul Health 2020;10:100553

34. de Robles P, Fiest KM, Frolkis AD, Pringsheim T, Atta C, St Germaine-Smith $C$, et al. The worldwide incidence and prevalence of primary brain tumors: a systematic review and meta-analysis. Neuro Oncol 2015;17:776-83. 